\title{
Prevalence of serum $N$-methyl-D-aspartate receptor autoantibodies in refractory psychosis
}

Katherine Beck, * John Lally, * Sukhwinder S. Shergill, Michael A. P. Bloomfield, James H. MacCabe, Fiona Gaughran** and Oliver D. Howes**

\begin{abstract}
Summary
N-methyl-D-aspartate receptor (NMDA-R) autoantibodies have been reported in people with acute psychosis. We hypothesised that their presence may be implicated in the aetiology of treatment-refractory psychosis. We sought to ascertain the point prevalence of NMDA-R antibody positivity in patients referred to services for treatment-refractory psychosis. We found that $3(7.0 \%)$ of 43 individuals had low positive NMDA-R antibody titres. This suggests that NMDA-R autoantibodies are unlikely
\end{abstract}

to account for a large proportion of treatment-refractory

\section{psychosis.}

\section{Declaration of interest}

F.G.: honoraria from Roche, BMS, Lundbeck and Sunovion; has a family member with professional links to Lilly and GSK. O.D.H.: investigator-initiated research funding from and/or advisory/speaker meetings organised by AstraZeneca, Eli Lilly, Jansenn, Lyden-Delta, Servier and Roche.
Autoimmune pathology has been suggested as an aetiology for some cases of schizophrenia, with increasing evidence implicating $\mathrm{N}$-methyl-D-aspartate receptor (NMDA-R) autoantibodies. ${ }^{1,2}$ NMDA-R hypofunction is postulated as a mechanism underlying the development of schizophrenia. ${ }^{3}$ Support for this theory comes from cases of NMDA-R encephalitis, which often presents with symptoms of psychosis before rapidly progressing to seizures, movement disorders, impaired consciousness, autonomic instability and respiratory distress. ${ }^{4}$ Previous studies have identified serum NMDA-R autoantibodies in $4.3 \%$ of a prospective cohort of patients with first-episode psychosis ${ }^{2}$ and $9.9 \%$ of acutely ill patients with schizophrenia - either in the first episode or experiencing an acute exacerbation of their illness. ${ }^{1}$ These studies indicate that $4-10 \%$ of patients in the first episode or acute phases of schizophrenia have NMDA-R autoantibodies. A recent study found that $10.5 \%$ of participants were seropositive for NMDA-R antibodies with no difference in seroprevalence between patients with schizophrenia and healthy controls. ${ }^{5}$ The same authors demonstrated in animal studies that NMDA-R autoantibodies have behavioural effects only in rodents with a compromised blood-brain barrier. This led them to hypothesise that it is a disruption of blood-brain barrier integrity in schizophrenia that leads to NMDA-R autoantibodies being able to enter the brain to a greater extent than in healthy controls. About one in three patients with schizophrenia has a treatment-refractory condition - defined by the National Institute of Health and Care Excellence (NICE) as persistent psychotic symptoms despite at least two adequate therapeutic trials of different antipsychotic drugs. ${ }^{6}$ In contrast to the dopamine elevation seen in patients who respond to treatment, patients with treatment- refractory psychosis have been found to have normal dopamine synthesis capacity ${ }^{7}$ and elevated glutamate levels. ${ }^{8}$ These findings are consistent with NMDA-R hypofunction, leading to compensatory glutamate excess in treatment-refractory illness. This would explain the lack of response to dopamine-blocking antipsychotic drugs. From this evidence, we hypothesised that NMDA-R hypofunction secondary to NMDA-R autoantibodies underlie some cases of treatmentrefractory psychosis. We sought to test this by ascertaining the point prevalence of NMDA-R antibody positivity in a sample of patients with treatment-refractory psychosis.

*These authors contributed equally to the work.

**Joint supervising authors.

\section{Method}

The sample consisted of 43 referrals (January 2011 to August 2013) to the services for treatment-refractory psychosis at South London and Maudsley NHS Foundation Trust. Routine sociodemographic and clinical data were collected as part of the assessment. These related to gender, age, ethnicity, diagnosis, duration of illness, response to treatment and NMDA-R autoantibody status. Inclusion criteria were meeting the ICD-10 criteria for schizophrenia (F20) or schizoaffective disorder (F25), ${ }^{9}$ and meeting the following criterion for treatment resistance: ${ }^{6}$ persistent psychotic symptoms associated with distress and functional impairment that have not responded to at least two adequate trials of different antipsychotics. An adequate treatment trial was defined as treatment at a dose within the British National Formulary (www.bnf.org) therapeutic range for at least 6 weeks, with good adherence indicated by receipt of a long-acting injection as prescribed, or, for oral antipsychotics, a serum antipsychotic level within the therapeutic range.

The primary outcome measure was seropositivity for NMDA-R antibodies. A standardised cell-based assay was used for the detection of serum immunoglobulin (IgG) antibodies directed against the NR1 and NR2b subunits of the NMDA-R. ${ }^{10}$ This was performed by the Department of Clinical Neurology, John Radcliffe Hospital, University of Oxford. Study approval was granted by the Psychosis Clinical Academic Group Audit committee at South London and Maudsley NHS Foundation Trust, London UK. Every effort has been made to preserve the anonymity of the patients in the case reports.

\section{Results}

The sample comprised 43 patients ( 32 males and 11 females; mean age 40.3 years (s.d. $=11.1$, range 20-69); schizophrenia, $n=36$; schizoaffective disorder, $n=7)$. The mean duration of illness was 15.7 years (s.d. $=9.4$, range $2-37$ ) and all met criteria for refractory illness. ${ }^{6}$ Three patients were seropositive for IgG antibodies directed against NMDA-R - giving a point prevalence of $7.0 \%$. All had low serum antibody titres $(1: 50,1: 50,1: 100)$. All three had normal MRI brain imaging and none had a history of delirium, neurological symptoms or signs, seizures or carcinoma. None of the patients had an electroencephalogram or cerebrospinal fluid (CSF) analysis.

The first patient was a 30-year-old White man, with a 10-year history of treatment-refractory paranoid schizophrenia, with a 
serum NMDA-R antibody titre of 1:50. The unremitting psychotic illness was characterised by grandiose and religious delusions, perceptual disturbances and conceptual disorganisation, as well as negative symptoms such as poor self-care and social withdrawal. His Brief Psychiatric Rating Scale-18 (BPRS-18) ${ }^{11}$ score at the time of serum antibody testing was 63 . The positive antibody result was identified concurrent to ongoing clozapine treatment. He displayed a good clinical response to maintenance clozapine therapy - reflected in a reduction in the BPRS-18 score to 30 after 6 months of treatment. A repeat NMDA-R antibody test at 6 months after the initial assay was negative.

The second patient was a 31-year-old Black British man with a 3 -year history of treatment-refractory paranoid schizophrenia. $\mathrm{He}$ had a serum NMDA-R antibody titre of 1:100. His initial presentation was marked by disorganisation of thought processes, delusions of reference and perceptual disturbances - including visual and command auditory hallucinations. The illness course was characterised by persistent positive psychotic symptoms. He had a BPRS-18 score of 52 on initial assessment, when he was treated with paliperidone palmitate. A second serum titre taken 5 weeks after his first positive result was 1:50 indicating a reduction in antibody levels without any noticeable change in clinical presentation. He showed a good clinical response to clozapine, demonstrated in a reduced BPRS-18 score of 31 after 5 months of clozapine treatment.

The third patient was a 41-year-old White woman. She had a serum NMDA-R antibody titre of 1:50. She had a diagnosis of treatment-refractory paranoid schizophrenia, with an illness duration of 16 years. Her illness was characterised by unremitting olfactory and tactile hallucinations. She had a BPRS-18 score of 71. She had a history of head injury with loss of consciousness but without sequelae. At the time of the antibody assay she was treated with quetiapine. She declined a change to her medication and her psychosis persists.

\section{Discussion}

This study found that 3 out of 43 patients (7.0\%) with chronic refractory psychosis were positive for NMDA-R autoantibodies. These results do not support our hypothesis that NMDA-R autoantibodies specifically underlie treatment-refractory schizophrenia, although research in larger samples is needed. Given recent evidence suggesting that seropositivity occurs in the acute phase of illness for those with chronic psychoses, ${ }^{1}$ it is possible that some of the seronegative patients with chronic refractory psychosis were seropositive for NMDA-R autoantibodies earlier in their illness. It is also possible that antipsychotic treatment may have decreased antibody titres. Thus, we cannot exclude the possibility that the patients had NMDA-R autoantibodies earlier in their illness, and that the effects of this persisted after NMDA-R antibody production had ceased. In all three of the participants who were seropositive, the antibody titres were low. The clinical significance of low titres in patients presenting with refractory psychosis and without the characteristic clinical syndrome remains to be determined. Although CSF and, to a lesser extent, serum antibody titres have been shown to be related to illness severity in NMDA-R encephalitis, ${ }^{12}$ a similar correlation has not yet been demonstrated in any stage of psychosis. In this study only serum samples were tested for the presence of NMDA-R antibodies, and it may be that the sensitivity of antibody testing could have been increased through the use of CSF. ${ }^{12}$ This assertion remains controversial, ${ }^{10}$ and has not been assessed in a population of individuals with treatment-refractory schizophrenia.

Limitations of our study were the relatively small sample size, the lack of a control group and the preponderance of men in our sample. The latter may be important, given evidence from studies of NMDA-R encephalitis indicating that women are more likely to be seropositive. ${ }^{4}$ Nevertheless, the excess of men in our sample is typical of refractory schizophrenia. ${ }^{6}$

Our study findings do not support the hypothesis that NMDA-R autoantibodies are a common aetiology in refractory psychotic illnesses, although we cannot exclude a role for them in the pathoaetiology of a subgroup of individuals. At present, there is no evidence that this is a useful routine investigation in refractory psychosis. However, further investigation is needed, given that there is evidence that some people with psychosis respond to immunomodulatory therapy. ${ }^{10}$

\section{Katherine Beck, MRCPsych, John Lally, MRCPsych, Sukhwinder S. Shergill, MBBS, FRCPsych, PhD, Michael A. P. Bloomfield, MRCPsych, James H. Maccabe MRCPsych, PhD, Department of Psychosis Studies, Institute of Psychiatry, King's College London; Fiona Gaughran, FRCP(I), FRCP, FRCPsych, MD, National Psychosis Service, South London and Maudsley NHS Foundation Trust, London; Oliver D. Howes, MRCPsych, PhD, DM, Department of Psychosis Studies, Institute of Psychiatry, King's College London, UK}

Correspondence: Oliver D. Howes, Institute of Psychiatry, Box 67, Camberwell, London SE5 8AF, UK. Email: oliver.howes@kcl.ac.uk

First received 30 Nov 2013, final revision 10 May 2014, accepted 1 Aug 2014

\section{Funding}

This study was funded by a Medical Research Council (UK) grant to O.D.H. (grant number: MC-A656-5QD30) and the National Institute of Health Research Biomedical Research Council grant to King's College London.

\section{Acknowledgements}

We thank Professor A. Vincent for assistance in analysis and interpretation of the results.

\section{References}

1 Steiner J, Walter M, Glanz W, Sarnyai Z, Bernstein HG, Vielhaber S, et al. Increased prevalence of diverse $\mathrm{N}$-methyl-D-aspartate glutamate receptor antibodies in patients with an initial diagnosis of schizophrenia: specific relevance of IgG NR1a antibodies for distinction from N-methyl-D-aspartate glutamate receptor encephalitis. JAMA Psychiatry 2013; 70: 271-8.

2 Zandi MS, Irani SR, Lang $B$, Waters $P$, Jones $P B$, McKenna $P$, et al. Disease-relevant autoantibodies in first episode schizophrenia. $J$ Neurol 2011; 258: 686-8.

3 Papanastasiou E, Stone JM, Shergill S. When the drugs don't work: the potential of glutamatergic antipsychotics in schizophrenia. Br J Psychiatry 2013; 202: 91-3.

4 Dalmau J, Gleichman AJ, Hughes EG, Rossi JE, Peng X, Lai M, et al. AntiNMDA-receptor encephalitis: case series and analysis of the effects of antibodies. Lancet Neurol 2008; 7:1091-8.

5 Hammer C, Stepniak B, Schneider A, Papiol S, Tantra M, Begemann M, et al. Neuropsychiatric disease relevance of circulating anti-NMDA receptor autoantibodies depends on blood-brain barrier integrity. Mol Psychiatry 2013; 3: 110.

6 Howes OD, Vergunst F, Gee S, McGuire P, Kapur S, Taylor D. Adherence to treatment guidelines in clinical practice: study of antipsychotic treatment prior to clozapine initiation. Br J Psychiatry 2012; 201: 481-5.

7 Demjaha A, Murray RM, McGuire PK, Kapur S, Howes OD. Dopamine synthesis capacity in patients with treatment-resistant schizophrenia. Am J Psychiatry 2012; 169: 1203-10.

8 Demjaha A, Egerton A, Murray RM, Kapur S, Howes OD, Stone JM, et al. Antipsychotic treatment resistance in schizophrenia associated with elevated glutamate levels but normal dopamine function. Biol Psychiatry 2014; 75: e11-3.

9 World Health Organization. The ICD-10 Classification of Mental and Behavioural Disorders: Clinical Descriptions and Diagnostic Guidelines. WHO, 1992.

10 Irani SR, Bera K, Waters P, Zuliani L, Maxwell S, Zandi MS, et al. N-methyl-Daspartate antibody encephalitis: temporal progression of clinical and paraclinical observations in a predominantly non-paraneoplastic disorder of both sexes. Brain 2010; 133: 1655-67.

11 Pull CB, Overall JE. Adequacy of the Brief Psychiatric Rating Scale for distinguishing lesser forms of psychopathology. Psychol Rep 1977; 40: 167-73.

12 Gresa-Arribas N, Titulaer MJ, Torrents A, Aguilar E, Mccracken L, Leypoldt F, et al. Antibody titres at diagnosis and during follow-up of anti-NMDA receptor encephalitis: a retrospective study. Lancet Neurol 2014; 13: 167-77. 